\title{
Impact of Trypanosoma cruzi on antimicrobial peptide gene expression and activity in the fat body and midgut of Rhodnius prolixus
}

\author{
CS Vieira ${ }^{1}$, PJ Waniek1 , DP Castro ${ }^{1}$, DP Mattos ${ }^{2}$, OC Moreira ${ }^{4}$ and P Azambuja ${ }^{1,3^{*}}$
}

\begin{abstract}
Background: Rhodnius prolixus is a major vector of Trypanosoma cruzi, the causative agent of Chagas disease in Latin America. In natural habitats, these insects are in contact with a variety of bacteria, fungi, virus and parasites that they acquire from both their environments and the blood of their hosts. Microorganism ingestion may trigger the synthesis of humoral immune factors, including antimicrobial peptides (AMPs). The objective of this study was to compare the expression levels of AMPs (defensins and prolixicin) in the different midgut compartments and the fat body of $R$. prolixus infected with different $T$. cruzi strains. The T. cruzi Dm 28c clone (Tcl) successfully develops whereas $Y$ strain (Tcll) does not complete its life- cycle in $R$. prolixus. The relative AMP gene expressions were evaluated in the insect midgut and fat body infected on different days with the T. cruzi Dm 28c clone and the $Y$ strain. The influence of the antibacterial activity on the intestinal microbiota was taken into account.
\end{abstract}

Methods: The presence of $T$. cruzi in the midgut of R. prolixus was analysed by optical microscope. The relative expression of the antimicrobial peptides encoding genes defensin (defA, defB, defC) and prolixicin (prol) was quantified by RT-qPCR. The antimicrobial activity of the AMPs against Staphylococcus aureus, Escherichia coli and Serratia marcescens were evaluated in vitro using turbidimetric tests with haemolymph, anterior and posterior midgut samples. Midgut bacteria were quantified using colony forming unit (CFU) assays and real time quantitative polymerase chain reaction (RT-qPCR).

Results: Our results showed that the infection of $R$. prolixus by the two different $T$. cruzi strains exhibited different temporal AMP induction profiles in the anterior and posterior midgut. Insects infected with T. cruzi Dm 28c exhibited an increase in defC and prol transcripts and a simultaneous reduction in the midgut cultivable bacteria population, Serratia marcescens and Rhodococcus rhodnii. In contrast, the T. cruzi Y strain neither induced AMP gene expression in the gut nor reduced the number of colony formation units in the anterior midgut. Beside the induction of a local immune response in the midgut after feeding $R$. prolixus with $T$. cruzi, a simultaneous systemic response was also detected in the fat body.

Conclusions: $R$. prolixus AMP gene expressions and the cultivable midgut bacterial microbiota were modulated in distinct patterns, which depend on the T. cruzi genotype used for infection.

Keywords: Rhodnius prolixus, Trypanosoma cruzi, Immune system, Antimicrobial peptides, Antibacterial activity, Microbiota

\footnotetext{
* Correspondence: azambuja.p@gmail.com

'Laboratório de Bioquímica e Fisiologia de Insetos, Instituto Oswaldo Cruz,

Fundação Oswaldo Cruz (IOC/FIOCRUZ), Rio de Janeiro, RJ, Brazil

${ }^{3}$ Departamento de Entomologia Molecular, Instituto Nacional de

Entomologia Molecular (INCT-EM), Rio de Janeiro, RJ, Brazil

Full list of author information is available at the end of the article
} 


\section{Background}

Trypanosoma cruzi is a protozoan parasite transmitted to vertebrate hosts by triatomine insects and is the causative agent of Chagas disease $[1,2]$. This disease is a public health problem, and it is estimated that approximately 6 to 7 million people are infected with $T$. cruzi worldwide, mostly in Latin America [3]. Rhodnius prolixus has been considered one of the most efficient $T$. cruzi vector in South America [2, 4]. Consequently, the medical importance of this species has stimulated studies of its physiology, immunology and molecular biology, especially in experimental infections with $T$. cruzi [5-12]. Other important reasons to study $R$. prolixus include its rapid developmental cycle and ease of colonization in laboratories compared to other triatomine species [13].

$T$. cruzi exhibits a variety of genotypes with a wide range of heterogeneous populations that circulate through vertebrate and invertebrate hosts [14, 15]. Many morphological, physiological and ecological variations of this parasite, including its infectivity and pathogenicity [16-18], may explain the various clinical manifestations of Chagas disease observed in different geographic regions [19]. Currently, the intraspecific nomenclature of $T$. cruzi is based on grouping populations into six discrete typing units (DTUs) from TcI to TcVI [20].

The various $T$. cruzi genotypes differ in their success at developing inside the digestive tract of different triatomine species [21, 22]. Previous studies have demonstrated that the T. cruzi Y strain, classified as TcII, cannot colonize the gut of $R$. prolixus, while the $T$. cruzi Dm 28c clone, classified as TcI, successfully infects $R$. prolixus [20, 23, 24]. Many factors intrinsic to the invertebrate host have been linked to parasite development, including the activation of humoral immune responses and the influence of natural gut bacterial microbiota [22, 23, 25].

Humoral immunity in insects is composed of a number of effector molecules that are rapidly synthesized after microorganism invasion. One important humoral response is the production of inducible antimicrobial peptides (AMPs) [26, 27]. AMPs are mainly synthesized by fat body cells and can diffuse into the haemolymph, which circulates around the entire insect body. Consequently, AMPs are able to control infection [28, 29]. AMPs are also produced in other insect tissues such as gut epithelial cells, where parasites might interact directly and induce the local synthesis and release of these molecules [30].

Similar to most animals, insects contain a rich natural gut microbiota, which is essential for diverse functions in the host such as digestion and vitamin production [30, 31]. These observations raise the important question of how insects manage AMP synthesis after parasite infection while maintaining the intestinal bacterial microbiota population. A previous study of the $R$. prolixus $-T$. cruzi interaction revealed that the infective $T$. cruzi Dm $28 \mathrm{c}$ clone causes a decrease in the cultivable gut bacteria, unlike the non-infective $T$. cruzi Y strain [10]. Moreover, differential T. cruzi susceptibility to lytic activity from the bacteria Serratia marcescens, which is commonly present in the midgut of $R$. prolixus, has been observed [23, 32]. These results suggest that the success of $T$. cruzi colonisation in the $R$. prolixus midgut depends on the parasite DTU and its capacity to interact with the natural vector microbiota. The aim of this study was to investigate the influence of $T$. cruzi infection on the spatial and temporal expression of antimicrobial peptides in $R$. prolixus and gut microbiota.

\section{Methods}

\section{Rhodnius prolixus maintenance and ethics statement}

$R$. prolixus were maintained in a colony at Laboratório de Bioquímica e Fisiologia de Insetos, Instituto Oswaldo Cruz, under controlled temperature and humidity. The insects were fed defibrinated rabbit blood provided by the Centro de Criação de Animais de Laboratório (Cecal) in an artificial apparatus [13]. The rabbit blood was obtained according to the Ethical Principles in Animal Experimentation approved by the Comissão de Ética no Uso de Animais do Instituto Oswaldo Cruz (CEUA/IOC) under the protocol number L-0061/08 developed by Conselho Nacional de Experimentação Animal/Ministério de Ciência e Tecnologia CONCEA/MCT [33].

\section{Trypanosoma cruzi culture}

The T. cruzi Dm 28c clone [34] and T. cruzi Y strain [35], previously classified as TcI and TcII, respectively [20], were maintained as epimastigote forms at $28{ }^{\circ} \mathrm{C}$ in brain heart infusion (BHI) media (Sigma-Aldrich) containing hemin and supplemented with $10 \%$ heat-inactivated bovine foetal serum [13]. For insect infection, the parasites were used in the exponential growth phase. The number of parasites was quantified in a Neubauer chamber using an optical microscope.

\section{Bacteria cultures}

Escherichia coli K12 4401 and Staphylococcus aureus 9518 were obtained from the National Collection of Industrial and Marine Bacteria (NCIMB), Aberdeen, UK. Serratia marcescens RPH was previously isolated from $R$. prolixus and maintained at Laboratório de Bioquímica $e$ Fisiologia de Insetos. All bacteria were kept at-70 ${ }^{\circ} \mathrm{C}$ in tryptone agar and $10 \%$ glycerol.

\section{Rhodnius prolixus oral infection}

Fifth instar nymphs were randomly chosen and fed with defibrinated rabbit blood containing $T$. cruzi epimastigotes of the Dm 28c clone or Y strain. The blood complement system was previously heat- inactivated by centrifugation 
at $1890 \times g$ for $15 \mathrm{~min}$ at $4{ }^{\circ} \mathrm{C}$ and incubation of the plasma (supernatant) for $30 \mathrm{~min}$ at $55{ }^{\circ} \mathrm{C}$. Subsequently, the plasma was mixed with phosphate buffered saline (PBS)-washed erythrocytes, and the parasites were added to the reconstituted blood at a final concentration of $1 \times 10^{7}$ epimastigotes $/ \mathrm{mL}$. Additionally, groups of insects received a blood meal containing a combination of two antibiotics (penicillin and ampicillin) with or without T. cruzi Dm 28c epimastigotes. Each antibiotic was administrated in a final concentration of $300 \mu \mathrm{g} / \mathrm{ml}$ of blood. Uninfected control insects were fed on inactivated blood without parasites. Only fully engorged fifth instar $R$. prolixus nymphs were used for the experiments.

\section{$T$. cruzi quantification in the $R$. prolixus midgut}

The entire digestive tract was dissected and individually homogenized in $1.0 \mathrm{~mL}$ PBS. The parasites were counted using a Neubauer haemocytometer as previously described [36].

\section{Quantification of the R. prolixus bacterial midgut microbiota (CFU)}

The anterior and posterior midgut were separately dissected from fifth instar nymphs infected or uninfected with $T$. $c r u z i(n=9)$. The cultivable microbiota population was quantified by counting the colony forming units (CFU) 7 days after feeding (DAF) as previously described [10]. Briefly, the midgut samples were serially tenfold diluted with sterile PBS, and $20 \mu \mathrm{L}$ aliquots of each dilution were spread on a Petri dish in sterile BHI agar (Sigma-Aldrich) culture medium. The plates were incubated at $30{ }^{\circ} \mathrm{C}$ for $24 \mathrm{~h}$, and the CFUs were quantified. As control, PBS was plated to check the sterility of the experiments. Additionally, RT-qPCR was performed using cDNA from 3 pools of 10 anterior midguts from different insect groups: control uninfected insects, treated with antibiotics (as described above), infected with T. cruzi Dm 28c; treated with antibiotics simultaneously infected with $T$. cruzi Dm 28c epimastigotes.

\section{Haemolymph and midgut antibacterial assays}

Anterior and posterior midguts were collected from dissected insects (nine pools of three insects each) and prepared as previously described [12, 36]. Haemolymph samples were pooled 5 DAF from 10 insects from three different experiments and diluted 1:1 in ultrapure water in sterile $1.5 \mathrm{ml}$ tubes containing a few crystals of phenylthiourea to avoid melanisation. The bacteria (S. aureus, $E$. coli and $S$. marcescens) were grown as previously described [36]. The antibacterial activity of the insect samples was assessed by modified turbidimetric assays (TB) [10]. All experiments were carried out at least in triplicate $(n=9)$. The detected antibacterial activities represent the
$R$. prolixus inducible humoral immune molecules and other factors produced by midgut microbiota.

\section{Quantification of antimicrobial peptide gene expression and intestinal bacteria by RT-qPCR}

Insects at 1 and 7 DAF ( $T$. cruzi infected and noninfected) were dissected to prepare three pools each of five anterior midguts, posterior midguts and fat body as previously described [12]. Total RNA was extracted using a NucleoSpin ${ }^{\odot}$ RNA II Kit (Macherey-Nagel, Düren, Germany) following the manufacturer's instructions and quantified using a NanoDrop 2000 Spectrophotometer (Thermo Scientific, Waltham, MA, USA). Synthesis of cDNA was carried out with a First-Strand cDNA Synthesis Kit (GE Healthcare, Buckinghamshire, UK) following the manufacturer's protocol using $2.5 \mu \mathrm{g}$ of total RNA and the $\operatorname{pd}(\mathrm{N})_{6}$ primer. cDNA was quantified by fluorescence using a Qubit Fluorimeter (Life Technologies) with the ssDNA assay kit. Real-time quantitative polymerase chain reactions (RT-qPCR) were conducted using an ABIPRISM 7500 Sequence Detection System (Applied Biosystems) at the PDTIS/FIOCRUZ facilities (Real-Time PCR Platform RPT-09A). The present study analysed the gene expression of three $R$. prolixus defensins (defA, $\operatorname{defB}$ and $\operatorname{defC})$ and prolixicin (prol). The specific primers for the AMP genes, $R$. prolixus reference genes and $16 \mathrm{~S}$ rRNA primers for relative quantification of $S$. marcescens and $R$. rhodnii were used as previously published or were designed based on the respective sequence (Additional file 1) [9, 37, 38], using the expression of control uninfected insects as calibrators. Each reaction was run in duplicate for each pool of insects $(n=3)$. Each well contained $10 \mathrm{ng}$ cDNA, primer pairs $(0.25 \mu \mathrm{M})$ and the qPCR master mix DyNAmo ColorFlash SYBR Green qPCR Kit (Thermo Fisher Scientific) at a final volume of $20 \mu \mathrm{l}$. The cDNA was amplified at $95{ }^{\circ} \mathrm{C}$ for $10 \mathrm{~min}$ followed by 40 cycles of $95^{\circ}$ for $15 \mathrm{~s}$ and $60{ }^{\circ} \mathrm{C}$ for $1 \mathrm{~min}$. As negative controls, PCR reactions were carried out without cDNA template to assess primer dimer formation or contamination in the reactions. A melting curve analysis was carried out to confirm that only a single product was amplified for each target. The AMP genes in the tissues of $R$. prolixus infected with $T$. cruzi were quantified by the comparative $\mathrm{Ct}(\Delta \Delta \mathrm{Ct})$ method [39] normalized with the $R$. prolixus reference genes $\alpha$-tubulin and GAPDH. Data were analysed by the Expression Suite v1.0.3 software (Life Technologies), considering the amplification efficiency of each target.

\section{Statistical analysis}

All obtained data were analyzed using Student's T-test and 1-way ANOVA and the GraphPad Prism 5 software. Significance levels are shown in the respective figures 


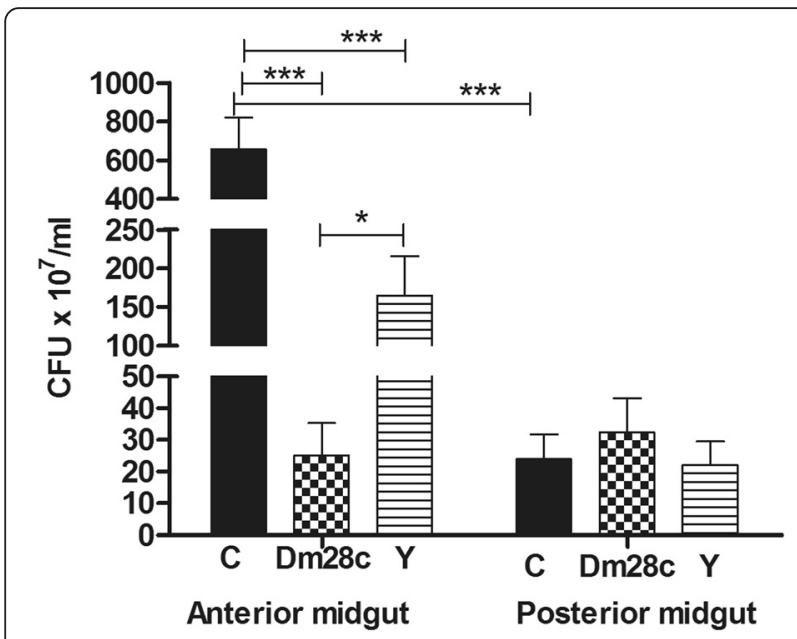

Fig. 1 Bacteria populations in the Rhodnius prolixus midgut infected with Trypanosoma cruzi. Colony forming units (CFU) were counted in the anterior and posterior midgut samples seven days after feeding the insects with blood at a final concentration of $1 \times 10^{7}$ epimastigotes $/ \mathrm{mL}$. Insect treatments: black columns-control insects (C); grid columns-T. cruzi Dm 28c clone-infected insects (Dm 28c); striped columns-T. cruzi $Y$ strain-infected insects $(Y)$. Bars represent the mean \pm SEM of three independent experiments with nine pools of insects $(n=9)$. Means were compared using Student's T-test or Mann-Whitney test; ${ }^{* * *} p<0.001,{ }^{*} p<0.05$

and legends, which were considered statistically different when $p<0.05$.

\section{Results}

\section{Quantification of $T$. cruzi in the $R$. prolixus midgut}

The parasite populations in the entire midgut of the $5^{\text {th }}$ instar $R$. prolixus nymphs were quantified from 2 to 7 days after feeding (DAF) with blood containing parasites. Two DAF, the average concentration of T. cruzi
Dm 28c $\left(75 \times 10^{4}\right.$ parasites $\left./ \mathrm{mL}\right)$ was ten times higher than that of T. cruzi Y $\left(7.5 \times 10^{4}\right.$ parasites $\left./ \mathrm{mL}\right)$ in the insect gut (Additional file $2, p<0.001$ ). On the $5^{\text {th }} \mathrm{DAF}$, a decrease in the number of parasites from both the $T$. cruzi strains was detected, although the T. cruzi Dm 28c number was two times greater than $T$. cruzi Y (Additional file $2, p<0.01)$. Moreover, no $T$. cruzi Y strain parasites were detected in the $R$. prolixus gut samples analysed on the $7^{\text {th }}$ DAF, while on the same day the $T$. cruzi Dm 28c population increased in all insect guts analysed (Additional file 2).

\section{Analysis of the $R$. prolixus midgut microbiota-colony forming units (CFU) and RT-qPCR}

The cultivable bacterial microbiota population in the $5^{\text {th }}$ instar nymphs infected with T. cruzi was evaluated separately for the anterior and posterior midgut using CFU counts. Infection with both $T$. cruzi strains significantly reduced the bacterial population in the $R$. prolixus anterior midgut on the $7^{\text {th }}$ DAF. However, T. cruzi Dm 28c caused a stronger CFU reduction $(p<0.001)(2.5 \times$ $10^{8} \mathrm{CFU} / \mathrm{mL}-26$-fold less) than $T$. cruzi Y $(p<0.05)$ $\left(1.64 \times 10^{9} \mathrm{CFU} / \mathrm{mL}-4\right.$-fold less $)$ in comparison with uninfected insects $\left(6.57 \times 10^{9} \mathrm{CFU} / \mathrm{mL}\right)$ (Fig. 1). T. cruzi infection did not significantly alter the CFU counts in the posterior midgut compared with control insects.

After T. cruzi Dm 28c infection a 2.6-fold reduction of the $S$. marcescens bacterial number was detected (Additional file $3 \mathrm{~A}, p<0.01$ ) and the $R$. rhodnii load decreased 3.5-fold (Additional file 3B, $p<0.01$ ) in the anterior midgut. In insects treated with antibiotics, $S$. marcescens was reduced 10-fold (Additional file 3A, $p<0.001$ ) whereas the $R$. rhodnii population increased 3-fold (Additional file $3 \mathrm{~B}, p<0.001)$. Data were always compared to control insects fed only on blood.
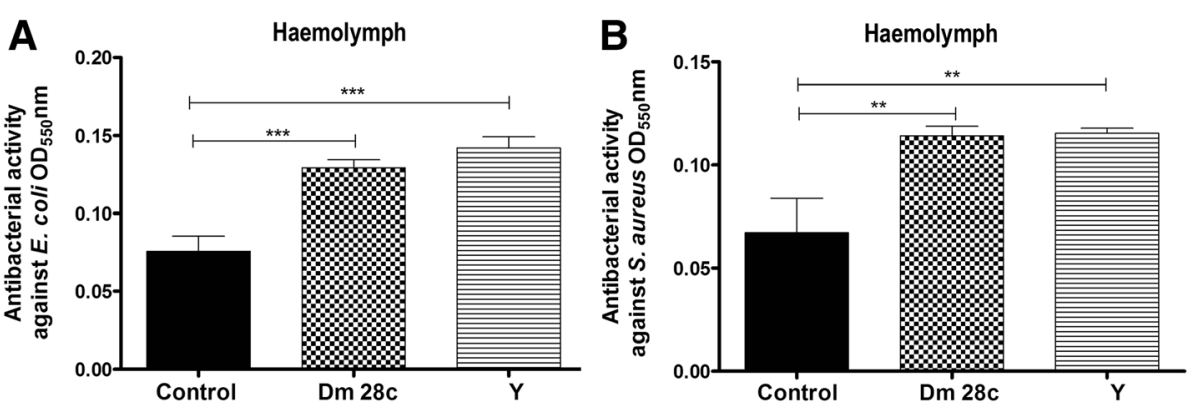

Fig. 2 Antibacterial activities in haemolymph samples of Rhodnius prolixus infected with Trypanosoma cruzi. R. prolixus $5^{\text {th }}$ instar nymphs were fed inactivated blood containing the T. cruzi Dm $28 \mathrm{c}$ clone or $Y$ strain at a final concentration of $1 \times 10^{7}$ epimastigotes $/ \mathrm{mL}$. The antibacterial activities were assayed with haemolymph collected five days after infection and tested against (a) E. coli or (b) S. aureus. Haemolymph samples were prepared from: black columns-control, uninfected insects; grid columns-infected with T. cruzi Dm 28c; striped columns-infected with T. cruzi Y strain. Antibacterial activity was measured through optical densities using the turbidimetric assay $\left(\mathrm{OD}_{550} \mathrm{~nm}\right)$ after $19 \mathrm{~h}$ of incubation of haemolymph samples with bacteria. Bars represent the mean \pm SEM of three independent experiments with nine pools of insects $(n=9)$. Means were compared using one-way ANOVA and Student's T-test; ${ }^{* * *} p<0.001,{ }^{* *} p<0.01$ 


\section{Haemolymph and midgut antibacterial detection}

The $R$. prolixus haemolymph antibacterial activity was demonstrated using TB assays. The haemolymph of uninfected control insects demonstrated antibacterial activities that inhibited the growth of both Gram-negative and Gram-positive bacteria (Fig. 2). However, haemolymph from both the T. cruzi infected insect groups exhibited significantly higher antibacterial activity than the haemolymph samples from the control insects (Fig. 2a, $p<0.001$; Fig. 2b, $p<0.001$ ).

The optical densities of the $R$. prolixus anterior midgut incubated with different bacteria showed that only the $T$. cruzi Dm 28c infection significantly increased the antibacterial activity against $S$. marcescens (Fig. 3c, $p<0.001$ ). The activities measured against $E$. coli and $S$. aureus in insects infected with both $T$. cruzi strains were not statistically significant compared to the control insects (Fig. 3a, 3b).

Antibacterial assays of the $R$. prolixus posterior midgut samples demonstrated that only T. cruzi Dm 28c infection induced an increase in the antibacterial activity against $E$. coli (Fig. 4a, $p<0.01$ ). No differences were observed for the activities against $S$. aureus in insects infected with the two T. cruzi strains compared to the control insects in this same midgut compartment (Fig. 4b).

\section{Quantification of antimicrobial peptide gene expression (RT-qPCR)}

Modulations in the expression of AMP genes in the $R$. prolixus $5^{\text {th }}$ instar nymphs infected with T. cruzi Dm 28c and T. cruzi $\mathrm{Y}$ strain were verified in different tissues at 1 and 7 DAF. All data obtained here were compared to the gene expression in control insects, which was given the value 1.0 and is represented in the figures as horizontal dotted lines along the Y-axis. Expression of prol in both the T. cruzi Dm 28c and Y strains was significantly upregulated at the transcript level in the fat body on the $7^{\text {th }}$ DAF in comparison to control insects (Fig. 5a, $p<0.05)$. However, only the T. cruzi Dm 28c infection modulated prol expression in the $R$. prolixus midgut, with distinct patterns in the different compartments. In the anterior midgut, prol transcript levels were significantly lower in infected insects than in controls on the $1^{\text {st }}$ DAF (Fig. 5a, $p<0.001$ ). On the $7^{\text {th }}$ DAF, the prol transcript levels had increased by 2.5 -fold in this same midgut compartment of T. cruzi Dm 28c-infected insects (Fig. $5 \mathrm{~b}, p<0.05$ ). However, the opposite pattern was observed in the posterior midgut, where the prol expression was 10 -fold higher in the T. cruzi Dm 28cinfected insects on the $1^{\text {st }}$ DAF (Fig. 5c, $p<0.001$ ), while on the $7^{\text {th }}$ DAF the prol transcript levels decreased, reaching the same levels as in the control insects (Fig. 5c). Additionally, the T. cruzi Y strain-infected insects exhibited prol gene expression similar to control
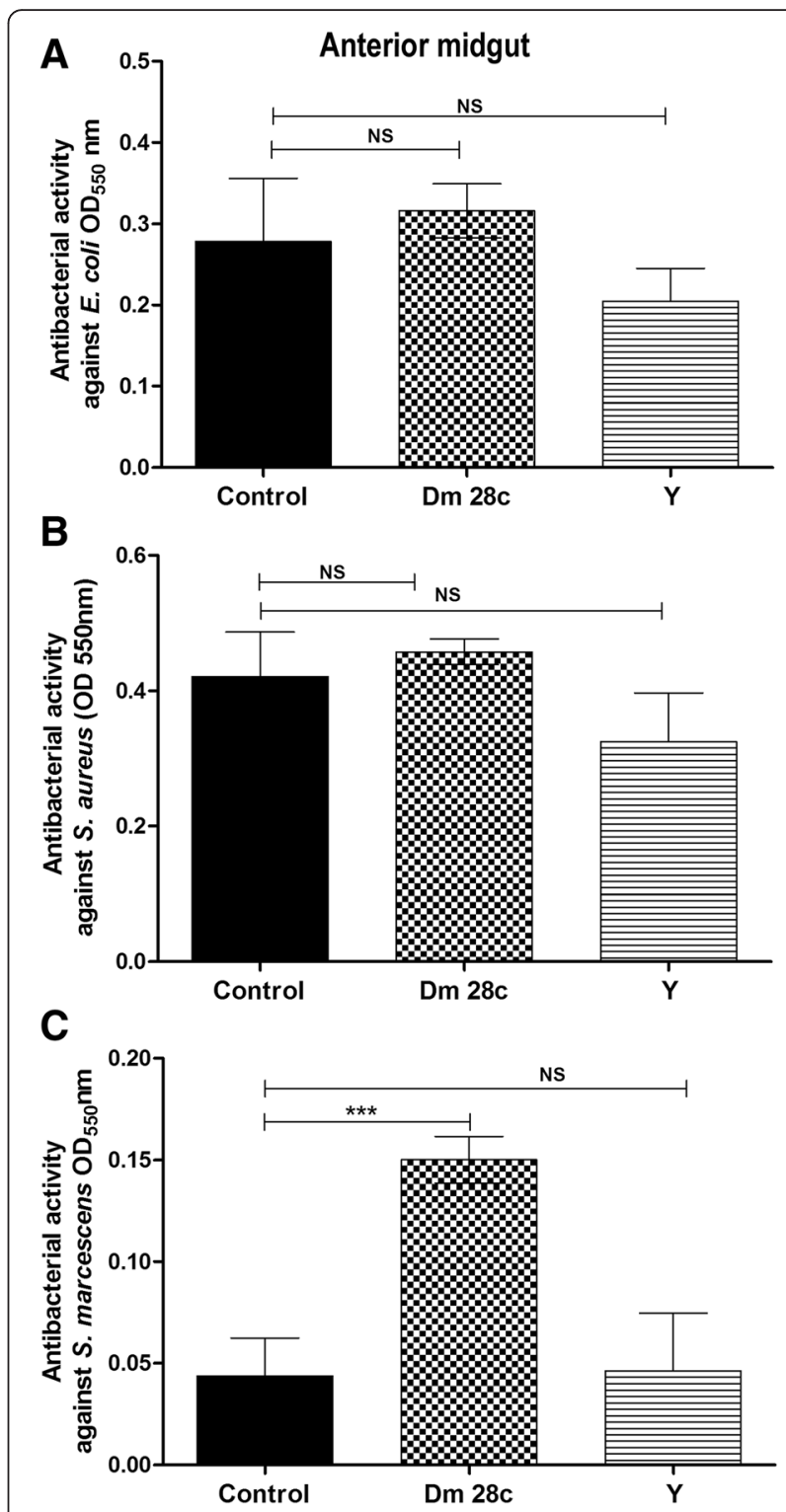

Fig. 3 Antibacterial activity in the anterior midgut of Rhodnius prolixus infected with Trypanosoma cruzi. R. prolixus $5^{\text {th }}$ instar nymphs were fed inactivated blood containing the T. cruzi Dm 28c clone or $Y$ strain at a final concentration of $1 \times 10^{7}$ epimastigotes $/ \mathrm{mL}$. The antibacterial activities were measured in vitro in anterior midgut samples seven days after infection and tested against (a) E. coli, (b) S. aureus and (c) S. marcescens. Treatments: black columns-uninfected insects; grid columns-T. cruzi Dm 28c-infected insects; striped columns-T. cruzi Y-infected insects. Antibacterial activity was measured through optical densities using the turbidimetric assay $\left(\mathrm{OD}_{550} \mathrm{~nm}\right)$ after $19 \mathrm{~h}$ of incubation of anterior midgut samples with bacteria. Bars represent the mean \pm SEM of three independent experiments with nine pools of insects $(n=9)$. Means were compared using one-way ANOVA and Student's $T$-test; ${ }^{* * *} p<0.001$, NS $=$ not significant

insects in both the anterior and posterior midgut on the $1^{\text {st }}$ and $7^{\text {th }}$ DAF (Fig. 5b, c).

Infection of $R$. prolixus with both the T. cruzi Dm 28c and Y strains down regulated the $\operatorname{def} A$ levels on the $1^{\text {st }}$ 

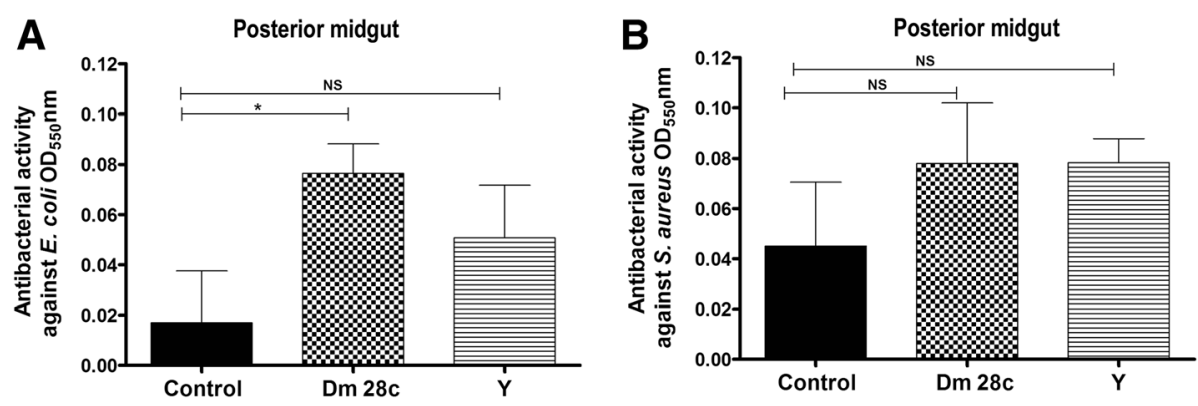

Fig. 4 Antibacterial activity in the posterior midgut of Rhodnius prolixus infected with Trypanosoma cruzi. R. prolixus $5^{\text {th }}$ instar nymphs were fed inactivated blood containing the T. cruzi Dm $28 \mathrm{c}$ clone or $Y$ strain at a final concentration of $1 \times 10^{7}$ epimastigotes $/ \mathrm{mL}$. The antibacterial activities were measured in vitro in posterior midgut samples seven days after infection and tested against (a) E. coli and (b) S. aureus. Treatments: black columns-uninfected insects; grid columns-T. cruzi Dm 28c-infected insects; striped columns-T. cruzi Y-infected insects. Antibacterial activity was measured through optical densities using the turbidimetric assay $\left(\mathrm{OD}_{550} \mathrm{~nm}\right)$ after $19 \mathrm{~h}$ of incubation of posterior midgut samples with bacteria. Bars represent the mean \pm SEM of three independent experiments with nine pools of insects $(n=9)$. Means were compared using one-way ANOVA and Student's T-test; ${ }^{*} p<0.05$

DAF in the fat body (Fig. 6a, $p<0.01$ ). On the $7^{\text {th }}$ DAF, a significant 2-fold increase was detected in the defA levels in the T. cruzi Dm 28c-infected insects (Fig. 6a, $p<0.05$ ), while the $T$. cruzi Y-infected insects presented the same gene levels as the control (Fig. 6a). In the anterior midgut, only the infection with $T$. cruzi Y modulated the defA transcript levels, which were significantly lower than in the control insects (Fig. 6b, $p<0.05$ ).

The ingestion of T. cruzi $\mathrm{Y}$ down regulated the $\operatorname{def} B$ levels on the $1^{\text {st }}$ DAF in the $R$. prolixus fat body and midgut (Fig. 6c, $p<0.05$; Fig. 6d, $p<0.01$ ). However, T. cruzi $\mathrm{Dm} 28 \mathrm{c}$ significantly upregulated $\operatorname{defB}$ levels in the anterior midgut, but only on the $1^{\text {st }}$ DAF (Fig. $6 \mathrm{~d}, p<0.05$ ). No $\operatorname{def} B$ transcripts were detected in the anterior midgut on the $7^{\text {th }}$ DAF for either of the T. cruzi strains (Fig. 6d).

Both $T$. cruzi strains strongly induced $\operatorname{defC}$ expression in the insect fat body on the $1^{\text {st }}$ DAF. While T. cruzi Dm $28 \mathrm{c}$ increased defC levels 11-fold (Fig. 7a, $p<0.001$ ), $T$. cruzi $\mathrm{Y}$ increased defC 24-fold (Fig. 7a, $p<0.001$ ) compared with non-infected insects. In the anterior and posterior midgut, only $T$. cruzi Dm 28c significantly upregulated $\operatorname{defC}$ levels on the $7^{\text {th }}$ DAF (Fig. $7 \mathrm{~b}, p<0.001$; Fig. $7 \mathrm{c}, p<0.05)$, whereas $T$. cruzi $\mathrm{Y}$ down regulated these genes in the posterior midgut on the $7^{\text {th }} \mathrm{DAF}$ (Fig. 7c, $p<0.01$ ).

In insects fed on blood containing antibiotics defC expression decreased 1.72-fold (Additional file 4, $p<0.05$ ) in the anterior midgut when compared to control noninfected insects. However, in insects treated with antibiotics and infected with $T$. cruzi Dm $28 \mathrm{c}$, defC expression was 4-fold higher (Additional file 4, $p<0.01$ ) in comparison to $T$. cruzi infected insects without antibiotics treatment and 14-fold higher than control, uninfected (Additional file $4, p<0.001)$. Summary of all results obtained are shown in Additional files 5 and 6.

\section{Discussion}

This study attempted to understand the influence of $T$. cruzi Dm 28c and T. cruzi Y infections on the modulation of AMP gene expression and antibacterial activity in different $R$. prolixus tissues, thereby altering the gut bacterial microbiota and parasite survival. It has already been demonstrated that $T$. cruzi Y strain is rapidly lysed, while T. cruzi Dm 28c complete its life-cycle in the gut of $R$. prolixus [10,23]. Comparing the infection of these two T. cruzi genotypes in $R$. prolixus, we found that Dm 28c induced:(i) a reduction of the CFU bacterial number, (ii) an increase of antibacterial activity against $S$. marcescens, (iii) an enhancement of prol and $\operatorname{defC}$ expression, and (iv) a decrease of $S$. marcescens and $R$. rhodnii load in the anterior midgut. In the present study, the insects fed on blood containing both antibiotics and T. cruzi Dm 28c, defC expression increased 4-fold in comparison to infected insects lacking antibiotics. In this context, a previous study demonstrated that $R$. prolixus fed on blood containing T. cruzi Dm 28c and antibiotics presented significantly increased parasite numbers and a reduced cultivable bacterial population in the midgut [10]. Together, all these findings suggest a regulatory function of defensin $\mathrm{C}$ on $R$. prolixus microbiota, promoting the reduction of intestinal bacteria number and subsequently the parasite development. S. marcescens - one cultivable bacteria commonly found in the triatomine's gut- has been associated with trypanolytic effects [23, 36, 40, 41]. This cytotoxic bacterium is a natural barrier that influences the establishment of parasites $[6,10,23]$. In contrast to Dm 28c, $T$. cruzi Y strain is apparently not able to stimulate the local immune response and overcome this barrier.

Antimicrobial effects of recombinant AMP prolixicin have been assayed against different Gram-negative bacterial species, showing higher activity against $E$. coli than 


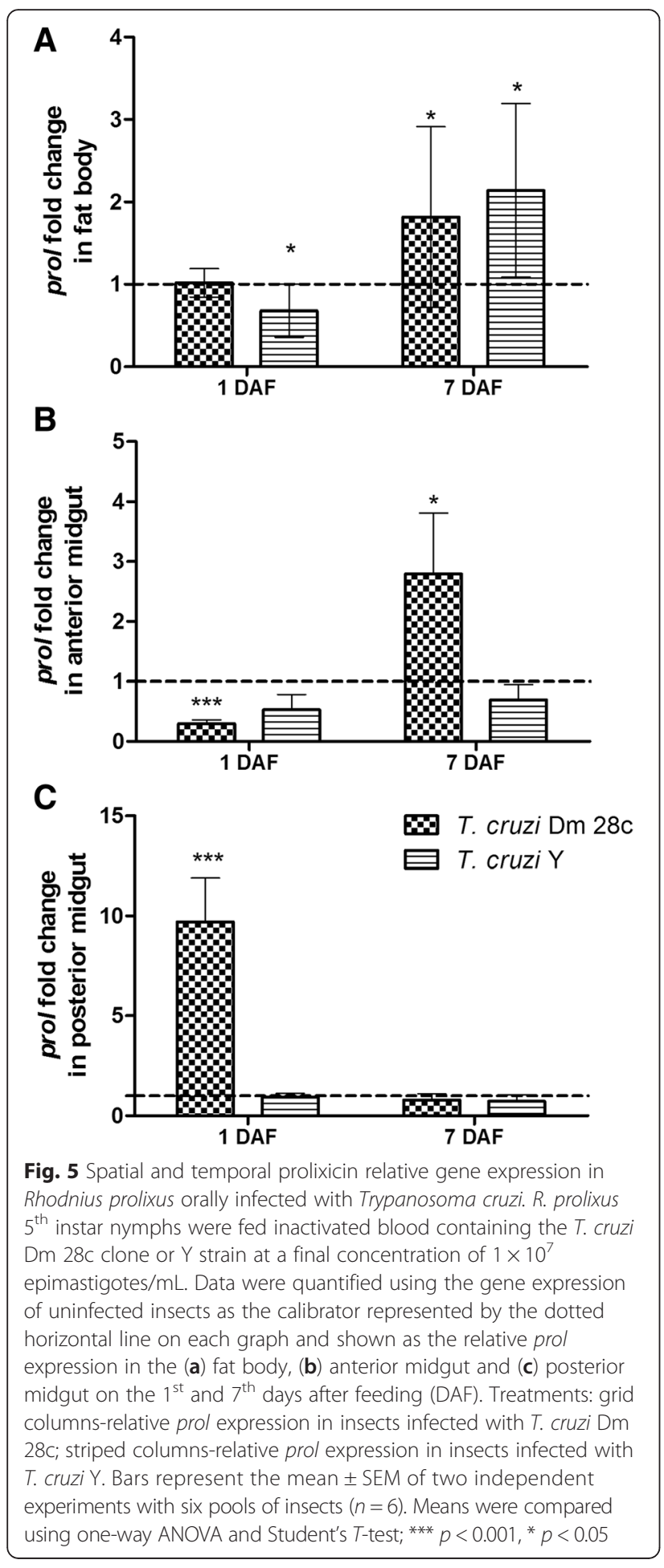

S. marcescens [9]. Here, the synergistic effects of prolixicin associated with the effects of defensin $C$ could explain the increased antibacterial activity against $S$. marcescens and the decrease of $R$. rhodnii and S. marcescens bacterial load in the anterior midgut after T. cruzi Dm 28c infection. On the other hand, $R$. prolixus infected with the T. cruzi strain Chile 5 did not exhibit a reduced $R$. rhodnii population [42]. These results suggest that the capacity of $T$. cruzi to colonize the $R$. prolixus midgut might depend on its genotypic characteristics combined with its ability to modulate (directly or indirectly) the host natural microbiota. In a recent study, the silencing of rpRelish (Nf-kB transcription factor of AMPs in the IMD pathway) resulted in an increase of $R$. rhodnii CFU, which did not affect T. cruzi Dm 28c clone development in $R$. prolixus midgut [43].

Many studies have described how the intestinal microbiota of insect vectors affect the life-cycle of parasites, including nutrient competition between these microorganisms [44, 45]. Native bacteria in the Anopheles midgut negatively affect certain species of Plasmodium by direct contact between the microorganisms involved and by the induction of the immune response mediated by commensal bacteria [46-48]. In contrast, parasitic infections capable of modulating immune peptide synthesis in insect hosts $[36,49,50]$ might interfere with the growth of certain bacteria species of the microbiota, as seems to occur in Trypanosoma rangeli infection in $R$. prolixus [36]. Moreover, serine protease inhibitors from the Kazal family in the anterior midgut of T. cruzi-infected $R$. prolixus were recently shown to be involved in the modulation of the intestinal microbiota [51].

The presence of bacteria or parasites in the digestive tract stimulates the systemic secretion of AMPs into the haemolymph of different insects, including species of Phlebotomus, Glossina and Drosophila, even without the invasion of these microorganisms into the haemocoel [49, 52-54]. After induction of AMP gene expression in the fat body, the respective peptides are initially secreted directly into the haemolymph. Later, the AMPs can diffuse throughout the insect body, representing a systemic response to oral parasite infection [29]. The induction of AMP expression in the fat body by parasites confined to the midgut could be due to immune signalling by molecules such as nitric oxide (NO), representing a host anticipation strategy to prevent a widespread infection [54, 55]. A similar pattern was observed in this study. The oral Infection of $R$. prolixus by both $T$. cruzi genotypes triggered a rapid increase of the $\operatorname{defC}$ gene transcriptional levels in the fat body. High levels of mature DefC in the fat body might be directly related to the increased antibacterial activity detected in vitro in the haemolymph.

During T. cruzi Dm 28c development in $R$. prolixus, higher numbers of epimastigotes are found in the anterior midgut until the fifth day after infection. Thereafter, they tend to inhabit the posterior midgut [56]. One day after T. cruzi Dm 28c infection, reduced prol levels were found in the anterior midgut, while prol levels had increased in posterior midgut. The T. cruzi $\mathrm{Y}$ infection was not capable of upregulating the expression of the 

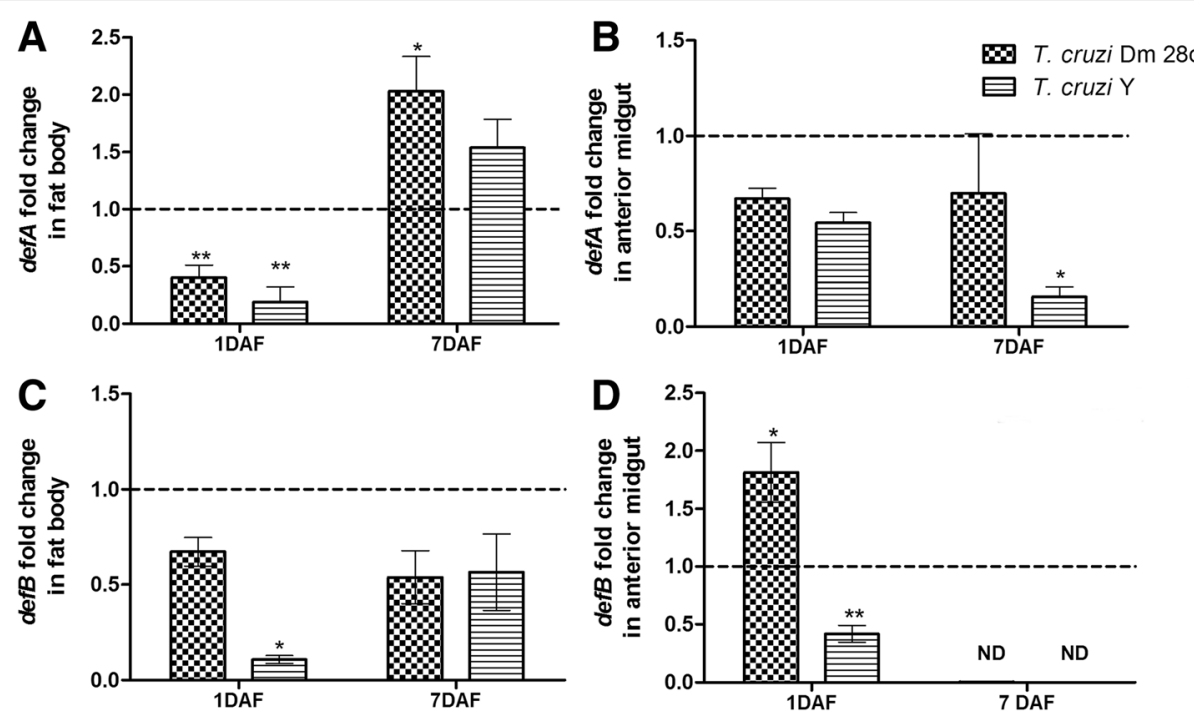

Fig. 6 Spatial and temporal defensin A and B relative gene expression in Rhodnius prolixus orally infected with Trypanosoma cruzi. R. prolixus $5^{\text {th }}$ instar nymphs were fed inactivated blood containing the T. cruzi Dm 28c clone or Y strain at a final concentration of $1 \times 10^{7}$ epimastigotes $/ \mathrm{mL}$. Data were quantified using the gene expression of uninfected insects as the calibrator, represented by the dotted horizontal line on each graph, and shown as the relative expression of (a) defA in the fat body, (b) defA in the anterior midgut, (c) defB in the fat body and (d) defB in the anterior midgut on the $1^{\text {st }}$ and $7^{\text {th }}$ days after feeding (DAF). Treatments: grid columns-relative gene expression in insects infected with $T$. cruzi Dm 28c; striped columns-relative gene expression in insects infected with T. cruzi Y. Bars represent the mean \pm SEM of two independent experiments with six pools of insects $(n=6)$. Means were compared using one-way ANOVA and Student's T-test; ${ }^{* *} p<0.01,{ }^{*} p<0.05$, ND $=$ not determined

analysed AMPs in the $R$. prolixus midgut. The expression of AMPs in intestinal epithelial cells is considered a local immune response activated by the direct contact of the parasites with the insect tissue [29, 57]. Here, the systemic response of $R$. prolixus to the different parasite strains was similar, although local responses to TCI and TcII infection exhibited differing profiles.

The parasite $P$. berghei modulates defensin gene expression in the gut and salivary gland epithelium of Anopheles gambiae, tissues where the parasite is found during its life-cycle [58]. Leishmania major infection also induces defensin expression in the haemolymph and midgut of the insect Phlebotomus duboscqi [49], although infection by Leishmania mexicana totally abolished def1 expression in Lutzomyia longipalpis [50]. Interestingly, the infection of $R$. prolixus with the T. rangeli Macias strain also upregulated $\operatorname{defC}$ levels in the midgut regions where the parasite was found [36]. In the present study, the presence of $T$. cruzi Dm $28 \mathrm{c}$ in different midgut regions had a similar effect, enhancing $\operatorname{defC}$ expression. These results support the hypothesis that defensin genes are modulated by protozoan infections in insects and not directly by the CFU bacterial microbiota population.

Several defensin-encoding genes have been identified in different triatomine species [11,37, 59-61]. After the analysis of the primary defensin structure and the transcript abundance of defensin-encoding genes in $T$. brasiliensis, it was proposed that these peptides have different biological targets [60]. In agreement, the three $R$. prolixus defensin encoding genes were differently expressed in response to the invading microorganism. In the present work, only $\operatorname{defC}$ was up regulated by $T$. cruzi Dm 28c, 7 DAF in the $R$. prolixus midgut. A similar result was also observed in $T$. rangeli infected insects [36]. On the other hand, $\operatorname{def} A$ and $\operatorname{def} B$ were upregulated in the midgut in response to Gram-positive bacterial oral infection [12].

Prolixicin was recently identified in $R$. prolixus haemolymph and shows structural similarities with another AMP family, the attacins [9]. The influence of attacins on the establishment of Trypanosoma brucei was previously demonstrated in the tsetse fly $[62,63]$. T. brucei infection upregulated the attacin genes in the fat body and midgut of the insect vector. In addition, insects naturally resistant to $T$. brucei infection present high levels of attacin even in non-infected flies, suggesting that this AMP interferes with T. brucei development $[62,63]$. Attacin and prolixicin possess high toxicity against the Gram-negative E. coli $[9,62]$, suggesting that the structural similarity between these AMPs may also extend to their microbial targets. Because prolixicin shares similar characteristics with attacins, this AMP may also have a toxic effect against trypanosomatids. The prolixicin gene expression seems to be downregulated according to the local concentration of $T$. cruzi epimastigotes, as observed in experimental infections with the $T$. rangeli Macias strain that successfully infects the $R$. prolixus midgut [36]. The 


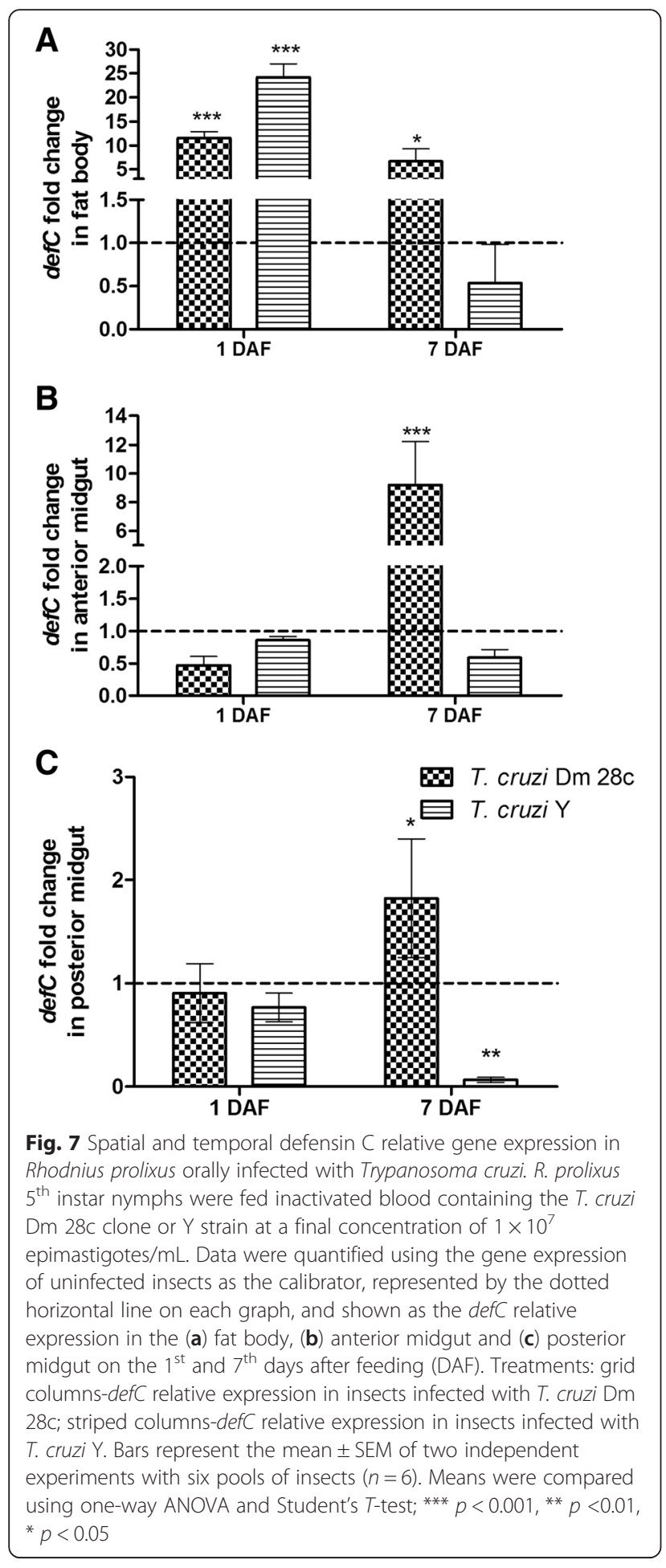

results obtained from $T$. cruzi infection in $R$. prolixus suggest that suppression of prol genes may represent one survival mechanism of trypanosomatids in the insect gut.

The genetic variability of T. cruzi is expressed in different compositions of cell surface molecules, such as sugars and proteins [64]. T. cruzi populations belonging to the $\mathrm{TcI}$ genotype contain more galactose residues in their cell surfaces than the TcII populations [65]. Membrane proteins such as the virulence factor transsialidase are also differently distributed in TcI and TcII genotypes [66]. Other membrane-bound proteins of $T$. cruzi, such as mucins, interact by the adhesion of the flagellum to the epithelial cell and perimicrovillar membranes of the midgut, a crucial step for parasite development and infectivity [67]. The role of transsialidases and mucins as virulent factors in the recognition of T. cruzi and in the induction of specific immune responses has been demonstrated in vertebrate models [68-70]. Therefore, the distinct immune response results obtained in the present study from $R$. prolixus infection by the two $T$. cruzi strains might be an effect of differences on T. cruzi membrane structures. Because R. prolixus is predominantly infected with TcI [71, 72], this genotype may be better adapted to this triatomine species due to a modulation of the immune response favouring the establishment of the parasite.

Currently, efforts to control human diseases transmitted by insects are focused on vector control, specifically in the construction of genetically modified intestinal bacteria that control their natural parasites by recombinant expression of an AMP or by RNAi [73, 74]. Knowledge about how different $T$. cruzi genotypes modulate antimicrobial peptide expression in $R$. prolixus can assist in selecting the genes to be manipulated. RNAi technology is an interesting tool to verify in the future the effect of each AMP gene expression on the parasite development and on the gut microbiota composition of $R$. prolixus. It would also be important to perform studies using aposymbiotic insects - which might have an effect on the triatomine immunity - to clarify the specific effects of each bacteria species and T. cruzi infections. However, these conditions would be artificial as in nature insects are frequently found inhabiting a great variability of bacterial species in the intestine and are infected with $T$. cruzi simultaneously [41]. To further clarify the tripartite relation between parasites, microbiota and insects, the measurement of protein levels and proteomic analyses will be useful in the future.

\section{Conclusions}

T. cruzi Dm 28c-infected $R$. prolixus showed increased $\operatorname{defC}$ and prol expression levels in the anterior midgut and a higher antibacterial activity against $S$. marcescens that could be related to the drastic reduction of the cultivable bacterial microbiota, S. marcescens and R. rhodnii. In contrast, these effects were not observed in insects infected with the T. cruzi Y strain, which might explain why this strain does not develop in $R$. prolixus. 


\section{Additional files}

Additional file 1: Oligonucleotide primers used for qPCR analysis. (DOC $46 \mathrm{~kb}$ )

Additional file 2: Parasite population in Rhodnius prolixus digestive tract The numbers of T. cruzi Dm $28 \mathrm{c}$ and $Y$ strain parasites were estimated in the whole digestive tracts of $R$. prolixus $5^{\text {th }}$ instar nymphs at different days after feeding (DAF). Each point represents the number of parasites in an individual insect, and bars indicate the median. Means were compared using Student's T-test or Mann-Whitney test;

*** $p<0.001,{ }^{*} p<0.05$. (BMP $64 \mathrm{~kb}$ )

Additional file 3: Determination of bacterial load in the anterior midgut of Rhodnius prolixus after antibiotic treatment and Trypanosoma cruzi Dm $28 \mathrm{c}$ infection by RT-qPCR. R. prolixus anterior midgut were analysed 7 days after feeding on inactivated blood containing antibiotics (ampicillin $300 \mu \mathrm{g} / \mathrm{ml}$ plus penicillin $300 \mu \mathrm{g} / \mathrm{ml}$ of blood) and T. cruzi Dm 28c clone at a final concentration of $1 \times 10^{7}$ epimastigotes $/ \mathrm{mL}$. Relative expression of 16S-rRNAS of A-Serratia marcescens B-Rhodococcus rhodnii. Treatments: black column — control insects fed only on blood; white column-insects fed on blood containing antibiotics; grid column-insects fed on blood containing T. cruzi; striped columns-insects fed on blood containing antibiotics and T. cruzi. Two biological samples in triplicate were used for each group. All data were normalized to the $R$. prolixus a-tubulin, representing the mean of identical triplicates \pm standard error. Bars represent the mean \pm SEM of 3 independent experiments with 3 pools of insects $(n=3)$. Means were compared using Student's T-test; ${ }^{* * *} p<0.001$, ** $p<0.01,{ }^{*} p<0.05$, NS indicates a non-significant difference. (TIF $244 \mathrm{~kb}$ )

Additional file 4: Defensin C relative gene expression in the anterior midgut of Rhodnius prolixus fed on blood containing antibiotics and Trypanosoma cruzi. DefC gene expression in $R$. prolixus anterior midgut were analysed 7 days after feeding on inactivated blood containing antibiotics (ampicillin $300 \mu \mathrm{g} / \mathrm{ml}$ plus penicillin $300 \mu \mathrm{g} / \mathrm{ml}$ of blood) and T. cruzi Dm 28c clone at a final concentration of $1 \times 10^{7}$ epimastigotes $/ \mathrm{mL}$. Treatments: black column-control insects fed only on blood; white columninsects fed on blood containing antibiotics; grid column-insects fed on blood containing T. cruzi; striped columns-insects fed on blood containing antibiotics and T. cruzi. Bars represent the mean \pm SEM of 3 independent experiments with 3 pools of insects $(n=3)$. Means were compared using Student's T-test; *** $p<0.001,{ }^{* *} p<0.01,{ }^{*} p<0.05$. (TIF $403 \mathrm{~kb}$ )

Additional file 5: Summary table of colony forming unit (CFU) and antibacterial activity against distinct Gram-negative and Gram-positive bacteria of $R$. prolixus midgut and haemolymph, 7 days after $T$. cruzi infection. (DOCX $12 \mathrm{~kb}$ )

Additional file 6: Summary table of antimicrobial peptides (AMP) gene expression in R. prolixus midgut and fat body, 1 and 7 days after T. cruzi infection. (DOCX $13 \mathrm{~kb}$ )

\section{Competing interests}

The authors declare that they have no competing interests.

\section{Authors' contributions}

Conceived and designed the experiments: CSV, PJW, DPC, OCM and PA. Carried out the biochemical experiments: CSV, DPM, DPC. Performed the molecular experiments: CSV, PJW, DPM and OCM. Analysis and interpretation of data: CSV, PJW, OCM and PA. Contributed reagents/materials: PA. Wrote the manuscript: CSV, PJW and PA. All authors read, corrected and approved the final manuscript.

\section{Acknowledgements}

This work was supported by grants from the Conselho Nacional de Desenvolvimento Científico e Tecnológico (CNPq), Fundação Oswaldo Cruz (FIOCRUZ) (PAPES and PROEP), Fundação de Amparo à Pesquisa do Estado do Rio de Janeiro (FAPERJ) and INCT-EM. The authors also thank the Program for Technological Development in Tools for Health-PDTIS/FIOCRUZ for use of the Real-Time PCR Platform RPT-09A facilities.

\section{Author details}

'Laboratório de Bioquímica e Fisiologia de Insetos, Instituto Oswaldo Cruz, Fundação Oswaldo Cruz (IOC/FIOCRUZ), Rio de Janeiro, RJ, Brazil.

${ }^{2}$ Laboratório deBiologia de Insetos, Universidade Federal Fluminense, Niterói, RJ, Brazil. ${ }^{3}$ Departamento de Entomologia Molecular, Instituto Nacional de Entomologia Molecular (INCT-EM), Rio de Janeiro, RJ, Brazil. ${ }^{4}$ Laboratório de Biologia Molecular e Doenças Endêmicas, Instituto Oswaldo Cruz, Fundação Oswaldo Cruz (IOC/FIOCRUZ), Rio de Janeiro, RJ, Brazil.

Received: 27 August 2015 Accepted: 20 February 2016

Published online: 01 March 2016

\section{References}

1. Chagas C. Nova tripanosomíase humana. Estudos sobre a morphologia e o ciclo evolutivo do Schizotrypanum cruzi, agente da nova entidade mórbida do homem. Mem Inst Oswaldo Cruz. 1909:1:159-218.

2. Coura JR. The main sceneries of Chagas disease transmission. The vectors, blood and oral transmissions-a comprehensive review. Mem Inst Oswaldo Cruz. 2015;110:277-82.

3. WHO. Chagas disease (American trypanosomiasis) Fact sheet $\mathrm{N}^{\circ} 340$. http://www.who.int/mediacentre/factsheets/fs340/en/. Accessed 22 Oct 2015.

4. Hashimoto K, Schofield CJ. Elimination of Rhodnius prolixus in central America. Parasit Vectors. 2012;5:45.

5. Wigglesworth $\vee$. The principles of insect physiology. 7th ed. New York: Chapman and Hall; 1982.

6. Azambuja P, Garcia ES, Ratcliffe NA. Gut microbiota and parasite transmission by insect vectors. Trends Parasitol. 2005;21:568-72.

7. Figueiredo MB, Castro DP, Nogueira NFS, Garcia ES, Azambuja P. Cellular immune response in Rhodnius prolixus: role of ecdysone in hemocyte phagocytosis. J Insect Physiol. 2006;52:711-6.

8. Garcia ES, Ratcliffe NA, Whitten MM, Gonzalez MS, Azambuja P. Exploring the role of insect host factors in the dynamics of Trypanosoma cruzi-Rhodnius prolixus interactions. J Insect Physiol. 2007;53:11-21.

9. Ursic-Bedoya R, Buchhop J, Joy JB, Durvasula R, Lowenberger C. Prolixicin: a novel antimicrobial peptide isolated from Rhodnius prolixus with differential activity against bacteria and Trypanosoma cruzi. Insect Mol Biol. 2011;20:775-86.

10. Castro D, Moraes C, Gonzalez M, Ratcliffe N, Azambuja P, Garcia E. Trypanosoma cruzi immune response modulation decreases microbiota in Rhodnius prolixus gut and is crucial for parasite survival and development. PLoS One. 2012;7, e36591.

11. Ribeiro JM, Genta FA, Sorgine MH, Logullo R, Mesquita RD, Paiva-Silva $\mathrm{GO}$, et al. An insight into the transcriptome of the digestive tract of the bloodsucking bug, Rhodnius prolixus. PLoS Negl Trop Dis. 2014;8, e2594.

12. Vieira CS, Waniek PJ, Mattos DP, Castro DP, Mello CB, Ratcliffe NA, et al. Humoral responses in Rhodnius prolixus: bacterial feeding induces differential patterns of antibacterial activity and enhances mRNA levels of antimicrobial peptides in the midgut. Parasit Vectors. 2014;7:232.

13. Azambuja P, Garcia ES. Care and maintenance of triatomine colonies. In Crampton JMBC, Louis C, editors. Molecular biology of insect disease vectors: a methods manual. London: Chapman and Hall; 1997. p. 56-64.

14. Araújo CAC, Waniek PJ, Jansen AM. An overview of Chagas disease and the role of triatomines on its distribution in Brazil. Vector Borne Zoonotic Dis. 2009;9:227-34.

15. Coura JR, Viñas PA. Chagas disease: a new worldwide challenge. Nature. 2010;465:S6-7.

16. Miles MA, Souza A, Povoa M, Shaw JJ, Lainson R, Toye PJ. Isozymic heterogeneity of Trypanosoma cruzi in the first autochthonous patients with Chagas' disease in Amazonian Brazil. Nature. 1978;272:819-21.

17. Miles MA, Lanham SM, de Souza AA, Povoa M. Further enzymic characters of Trypanosoma cruzi and their evaluation for strain identification. Trans R Soc Trop Med Hyg. 1980;74:221-37.

18. Miles MA, Llewellyn MS, Lewis MD, Yeo M, Baleela R, Fitzpatrick S, et al. The molecular epidemiology and phylogeography of Trypanosoma cruzi and parallel research on Leishmania: looking back and to the future. Parasitology. 2009:136:1509-28.

19. Miles MA, Cedillos RA, Povoa MM, de Souza AA, Prata A, Macedo V. Do radically dissimilar Trypanosoma cruzi strains (zymodemes) cause venezuelan and brazilian forms of Chagas' disease? Lancet. 1981;1:1338-40. 
20. Zingales B, Andrade SG, Briones MR, Campbell DA, Chiari E, Fernandes O, et al. A new consensus for Trypanosoma cruzi intraspecific nomenclature: second revision meeting recommends Tcl to TcVI. Mem Inst Oswaldo Cruz. 2009;104:1051-4.

21. Brener Z. Biology of Trypanosoma cruzi. Annu Rev Microbiol. 1973;27:347-82.

22. Garcia ES, Azambuja P. Development and interactions of Trypanosoma cruzi within the insect vector. Parasitol Today. 1991;7:240-4.

23. Azambuja P, Feder D, Garcia ES. Isolation of Serratia marcescens in the midgut of Rhodnius prolixus: impact on the establishment of the parasite Trypanosoma cruzi in the vector. Exp Parasitol. 2004;107:89-96.

24. Vallejo GA, Guhl F, Schaub GA. Triatominae-Trypanosoma cruzi/T. rangeli: vector-parasite interactions. Acta Trop. 2009;110:137-47.

25. Azambuja P, Feder D, Mello C, Gomes S, Garcia E. Immunity in Rhodnius prolixus: trypanosomatid-vector interactions. Mem Inst Oswaldo Cruz. 1999;94 Suppl 1:219-22.

26. Steiner $H$, Hultmark D, Engstrom A, Bennich $H$, Boman HG. Sequence and specificity of two antibacterial proteins involved in insect immunity. Nature. 1981;292:246-8.

27. Ferrandon D, Imler JL, Hetru C, Hoffmann JA. The Drosophila systemic immune response: sensing and signalling during bacterial and fungal infections. Nat Rev Immunol. 2007;7:862-74.

28. Lamberty M, Zachary D, Lanot R, Bordereau C, Robert A, Hoffmann JA, et al. Insect immunity. Constitutive expression of a cysteine-rich antifungal and a linear antibacterial peptide in a termite insect. J Biol Chem. 2001;276:4085-92.

29. Bulet $P$, Stocklin R. Insect antimicrobial peptides: structures, properties and gene regulation. Protein Pept Lett. 2005;12:3-11.

30. Charroux B, Royet J. Drosophila immune response: from systemic antimicrobial peptide production in fat body cells to local defense in the intestinal tract. Fly. 2010;4:40-7

31. Garcia ES, Genta FA, de Azambuja P, Schaub GA. Interactions between intestinal compounds of triatomines and Trypanosoma cruzi. Trends Parasitol. 2010:26:499-505.

32. Castro DP, Seabra SH, Garcia ES, de Souza W, Azambuja P. Trypanosoma cruzi: ultrastructural studies of adhesion, lysis and biofilm formation by Serratia marcescens. Exp Parasitol. 2007;117:201-7.

33. Laboratório SBdCdAe. COBEA. http://www.cobea.org.br. Accessed 22 Oct 2015.

34. Contreras VT, Araújo-Jorge TC, Bonaldo MC, Thomaz N, Barbosa HS, Meirelles Mde N, et al. Biological aspects of the Dm 28c clone of Trypanosoma cruzi after metacyclogenesis in chemically defined media. Mem Inst Oswaldo Cruz. 1988;83:123-33.

35. Silva LHP, Nussenzweig V. Sôbre uma cepa de Trypanosoma cruzi altamente virulenta para o camundongo branco. Folia Clin Biol. 1953;20:191-208.

36. Vieira CS, Mattos DP, Waniek PJ, Santangelo JM, Figueiredo MB, Gumiel $\mathrm{M}$, et al. Rhodnius prolixus interaction with Trypanosoma rangeli: modulation of the immune system and microbiota population. Parasit Vectors. 2015;8:135

37. Lopez L, Morales G, Ursic R, Wolff M, Lowenberger C. Isolation and characterization of a novel insect defensin from Rhodnius prolixus, a vector of Chagas disease. Insect Biochem Mol Biol. 2003;33:439-47.

38. Paim RM, Pereira MH, Di Ponzio R, Rodrigues JO, Guarneri AA, Gontijo NF, et al. Validation of reference genes for expression analysis in the salivary gland and the intestine of Rhodnius prolixus (Hemiptera, Reduviidae) under different experimental conditions by quantitative real-time PCR. BMC Res Notes. 2012;5:128.

39. Livak KJ, Schmittgen TD. Analysis of relative gene expression data using real-time quantitative PCR and the 2(-delta delta $C(T))$ method. Methods. 2001;25:402-8.

40. Da Mota FF, Marinho LP, Moreira CJ, Lima MM, Mello CB, Garcia ES, et al. Cultivation-independent methods reveal differences among bacterial gut microbiota in triatomine vectors of Chagas disease. PLoS Negl Trop Dis. 2012;6, e1631.

41. Gumiel M, da Mota FF, Rizzo Vde S, Sarquis O, de Castro DP, Lima MM, et al. Characterization of the microbiota in the guts of Triatoma brasiliensis and Triatoma pseudomaculata infected by Trypanosoma cruzi in natural conditions using culture independent methods. Parasit Vectors. 2015;8:245

42. Eichler S, Schaub GA. Development of symbionts in triatomine bugs and the effects of infections with trypanosomatids. Exp Parasitol. 2002;100:17-27.

43. Mesquita RD, Vionette-Amaral RJ, Lowenberger C, Rivera-Pomar R, Monteiro FA, Minx P et al. Genome of Rhodnius prolixus, an insect vector of Chagas disease, reveals unique adaptations to hematophagy and parasite infection. Proc Natl Acad Sci USA. 2015;12:14936-41.
44. Weiss B, Aksoy S. Microbiome influences on insect host vector competence. Trends Parasitol. 2011;27:514-22.

45. Gendrin M, Christophides G. The Anopheles mosquito microbiota and their impact on pathogen transmission. In: Manguin S, editor. Anopheles mosquitoes-New insights into malaria vectors. Croatia: InTech; 2013. p. 525-48.

46. Dong Y, Manfredini F, Dimopoulos G. Implication of the mosquito midgut microbiota in the defense against malaria parasites. PLoS Pathog. 2009;5, e1000423.

47. Meister S, Agianian B, Turlure F, Relogio A, Morlais I, Kafatos FC, et al. Anopheles gambiae PGRPLC-mediated defense against bacteria modulates infections with malaria parasites. PLoS Pathog. 2009;5, e1000542.

48. Cirimotich CM, Dong Y, Clayton AM, Sandiford SL, Souza-Neto JA, Mulenga $M$, et al. Natural microbe-mediated refractoriness to Plasmodium infection in Anopheles gambiae. Science. 2011;332:855-8.

49. Boulanger N, Lowenberger C, Volf P, Ursic R, Sigutova L, Sabatier L, et al. Characterization of a defensin from the sand fly Phlebotomus duboscai induced by challenge with bacteria or the protozoan parasite Leishmania major. Infect Immun. 2004:72:7140-6.

50. Telleria EL, Sant'Anna MR, Alkurbi MO, Pitaluga AN, Dillon RJ, Traub-Csekö YM. Bacterial feeding, Leishmania infection and distinct infection routes induce differential defensin expression in Lutzomyia longipalpis. Parasit Vectors. 2013;6:12

51. Soares TS, Buarque DS, Queiroz BR, Gomes CM, Braz GR, Araujo RN, et al A kazal-type inhibitor is modulated by Trypanosoma cruzi to control microbiota inside the anterior midgut of Rhodnius prolixus. Biochimie. 2015;112:41-8.

52. Boulanger $N$, Ehret-Sabatier L, Brun R, Zachary D, Bulet $P$, Imler JL. Immune response of Drosophila melanogaster to infection with the flagellate parasite Crithidia spp. Insect Biochem Mol Biol. 2001;31:129-37.

53. Boulanger N, Brun R, Ehret-Sabatier L, Kunz C, Bulet P. Immunopeptides in the defense reactions of Glossina morsitans to bacterial and Trypanosoma brucei brucei infections. Insect Biochem Mol Biol. 2002;32:369-75.

54. Hao Z, Kasumba I, Lehane MJ, Gibson WC, Kwon J, Aksoy S. Tsetse immune responses and trypanosome transmission: implications for the development of tsetse-based strategies to reduce trypanosomiasis. Proc Natl Acad Sci USA. 2001;98:12648-53.

55. Hao Z, Kasumba I, Aksoy S. Proventriculus (cardia) plays a crucial role in immunity in tsetse fly (Diptera: Glossinidiae). Insect Biochem Mol Biol. 2003;33:1155-64.

56. Cortez MR, Provencano A, Silva CE, Mello CB, Zimmermann LT, Schaub GA, et al. Trypanosoma cruzi: effects of azadirachtin and ecdysone on the dynamic development in Rhodnius prolixus larvae. Exp Parasitol. 2012;131:363-71.

57. Tzou P, Ohresser S, Ferrandon D, Capovilla M, Reichhart JM, Lemaitre B, et al. Tissue-specific inducible expression of antimicrobial peptide genes in Drosophila surface epithelia. Immunity. 2000;13:737-48.

58. Dimopoulos G, Seeley D, Wolf A, Kafatos FC. Malaria infection of the mosquito Anopheles gambiae activates immune-responsive genes during critical transition stages of the parasite life cycle. EMBO J. 1998;17:6115-23.

59. Araújo CAC, Waniek PJ, Stock P, Mayer C, Jansen AM, Schaub GA. Sequence characterization and expression patterns of defensin and lysozyme encoding genes from the gut of the reduviid bug Triatoma brasiliensis. Insect Biochem Mol Biol. 2006;36:547-60.

60. Waniek PJ, Castro HC, Sathler PC, Miceli L, Jansen AM, Araújo CAC. Two novel defensin-encoding genes of the Chagas disease vector Triatoma brasiliensis (Reduviidae, Triatominae): gene expression and peptide-structure modeling. J Insect Physiol. 2009;55:840-8.

61. Araújo CAC, Lima AC, Jansen AM, Galvão C, Jurberg J, Costa J, et al. Genes encoding defensins of important Chagas disease vectors used for phylogenetic studies. Parasitol Res. 2015;114:4503-11.

62. Hu Y, Aksoy S. An antimicrobial peptide with trypanocidal activity characterized from Glossina morsitans morsitans. Insect Biochem Mol Biol. 2005;35:105-15.

63. Hu C, Aksoy S. Innate immune responses regulate trypanosome parasite infection of the tsetse fly Glossina morsitans morsitans. Mol Microbiol. 2006:60:1194-204

64. De Souza W. Structural organization of the cell surface of pathogenic protozoa. Micron. 1995;26:405-30.

65. Araújo CAC, Mello CB, Jansen AM. Trypanosoma cruzi I and Trypanosoma cruzi Il: recognition of sugar structures by Arachis hypogaea (peanut agglutinin) lectin. J Parasitol. 2002;88:582-6. 
66. Burgos JM, Risso MG, Breniere SF, Barnabe C, Campetella O, Leguizamon MS Differential distribution of genes encoding the virulence factor trans-sialidase along Trypanosoma cruzi discrete typing units. PLoS One. 2013;8, e58967.

67. Gonzalez MS, Souza MS, Garcia ES, Nogueira NF, Mello CB, Canepa GE, et al. Trypanosoma cruzi TCSMUG L-surface mucins promote development and infectivity in the triatomine vector Rhodnius prolixus. PLoS Negl Trop Dis. 2013; , e2552.

68. Abel LC, Ferreira LR, Cunha Navarro I, Baron MA, Kalil J, Gazzinelli RT, et al. Induction of IL-12 production in human peripheral monocytes by Trypanosoma cruzi is mediated by glycosylphosphatidylinositol-anchored mucin-like glycoproteins and potentiated by IFN- $\gamma$ and CD40-CD40L interactions. Mediators Inflamm. 2014;2014:345659.

69. Freire-de-Lima L, Fonseca LM, Oeltmann T, Mendonça-Previato L, Previato JO. The trans-sialidase, the major Trypanosoma cruzi virulence factor: three decades of studies. Glycobiology. 2015:25:1142-9.

70. Schocker NS, Portillo S, Brito CR, Marques AF, Almeida IC, Michael K. Synthesis of gala $(1,3)$ gal $3(1,4) \mathrm{Gl}$ cNAca-, gal $3(1,4) \mathrm{GlcNAca-}$ and GlcNAca-containing neoglycoproteins and their immunological evaluation in the context of Chagas disease. Glycobiology. 2016;26:39-50.

71. Araújo CAC, Waniek PJ, Xavier SC, Jansen AM. Genotype variation of Trypanosoma cruzi isolates from different Brazilian biomes. Exp Parasitol. 2011:127:308-12.

72. Araújo CAC, Waniek PJ, Jansen AM. Tcl/Tcll co-infection can enhance Trypanosoma cruzi growth in Rhodnius prolixus. Parasit Vectors. 2014;7:94

73. Hurwitz I, Fieck A, Read A, Hillesland H, Klein N, Kang A, et al. Paratransgenic control of vector borne diseases. Int J Biol Sci. 2011;7:1334-44.

74. Taracena ML, Oliveira PL, Almendares O, Umana C, Lowenberger C, Dotson EM, et al. Genetically modifying the insect gut microbiota to control Chagas disease vectors through systemic RNAi. PLoS Negl Trop Dis. 2015;9, e0003358.

\section{Submit your next manuscript to BioMed Central and we will help you at every step:}

- We accept pre-submission inquiries

- Our selector tool helps you to find the most relevant journal

- We provide round the clock customer support

- Convenient online submission

- Thorough peer review

- Inclusion in PubMed and all major indexing services

- Maximum visibility for your research

Submit your manuscript at www.biomedcentral.com/submit

C Biomed Central 